\title{
Inhibition of human neutrophil oxidative burst by pyrazolone derivatives
}

\author{
David Costa $^{a}$, Alexandra P. Marques ${ }^{b}$, Rui L. Reis ${ }^{b}$, José L. F. C. Lima ${ }^{\text {a }}$, Eduarda Fernandes ${ }^{\mathrm{a}, *}$ \\ ${ }^{a}$ REQUIMTE, Departamento de Química-Física, Faculdade de Farmácia, Universidade do Porto, Rua Aníbal Cunha, 164, 4099-030 Porto, Portugal \\ b $3 B$ 's Research Group-Biomaterials, Biodegradables, Biomimetics, Department of Polymer Engineering, University of Minho, \\ Campus de Gualtar, 4710-057 Braga, Portugal
}

Received 6 September 2005; accepted 15 September 2005

Available online 21 October 2005

\begin{abstract}
The risk of agranulocytosis associated with the use of pyrazolone drugs at therapeutical doses and for short periods of time has been considered to be very low. However, little or no attention at all has been devoted to the possible hindrance of neutrophil burst and scavenging of neutrophilgenerated reactive oxygen species (ROS) by these compounds. Such an effect could be beneficial in the case of overactivation of neutrophils but could also be highly detrimental if the number of circulating neutrophils is already decreased. Thus, the aim of the present study was to evaluate the putative inhibitory effect of the pyrazolones dipyrone, aminopyrine, isopropylantipyrine, and antipyrine against human neutrophil burst and their scavenging activity against $\mathrm{O}_{2}{ }^{-}, \mathrm{H}_{2} \mathrm{O}_{2}, \mathrm{HO}^{\circ}$, ROO${ }^{*}$, and $\mathrm{HOCl}$. The obtained results showed that dipyrone and aminopyrine prevent phorbol-12-myristate-13-acetate-induced neutrophil burst with high efficiency, while isopropylantipyrine had little effect and antipyrine had no effect at all. Dipyrone and aminopyrine were highly potent scavengers of $\mathrm{HO}^{\circ}$ and $\mathrm{HOCl}$, while, in accordance with the neutrophil burst results, isopropylantipyrine had little effect and antipyrine had no effect at all against these two ROS. None of the studied pyrazolones was capable of scavenging $\mathrm{O}_{2}^{--}$or $\mathrm{H}_{2} \mathrm{O}_{2}$, while dipyrone was shown to be the most reactive against $\mathrm{ROO}^{\circ}$.
\end{abstract}

(C) 2005 Elsevier Inc. All rights reserved.

Keywords: Human neutrophils; Oxidative burst; Myeloperoxidase; NADPH oxidase; Reactive oxygen species; Pyrazolone derivatives; Dipyrone; Aminopyrine; Isopropylantipyrine; Antipyrine; Free radicals

\section{Introduction}

Neutrophils (polymorphonuclear leukocytes; PMNs) are the most abundant leukocytes of the blood and participate actively in the innate host defence response. During this response, PMNs and other phagocytic cells are mobilized to sites of injury or infection where they ingest and kill invading microorganisms [1]. These are also the first type of cells to arrive at an inflammatory site where they play a major role in the inflammation and tissue damage of noninfectious diseases such as arthritis, inflammatory bowel disease, and ischemiareperfusion injury [2].

PMNs use an extraordinary array of oxygen-dependent and oxygen-independent microbicidal weapons to destroy and remove infectious agents. Oxygen-dependent mechanisms involve the production of reactive oxygen species (ROS) and

\footnotetext{
* Corresponding author. Fax: +351 222004427.

E-mail address: egracas@ff.up.pt (E. Fernandes).
}

oxygen-independent mechanisms include most other neutrophil functions, such as chemotaxis, phagocytosis, degranulation, and release of lytic enzymes and bactericidal peptides [3]. The generation of ROS from stimulated neutrophils is thought to play an important role in host defence and tissue damage $[4,5]$. When neutrophils are activated on contact with soluble stimuli or by ingestion of foreign materials into phagosomes (phagocytosis), they initiate a "respiratory burst" by consuming molecular oxygen $\left(\mathrm{O}_{2}\right)$, resulting in the formation of superoxide radical $\left(\mathrm{O}_{2}^{\cdot-}\right)$ via the action of a plasma-membrane NADPH oxidase $[3,6]$. The function of NADPH oxidase in PMNs is thus to provide ROS that kill organisms. $\mathrm{O}_{2}^{{ }^{--}}$is important as the primary product for the neutrophil-induced generation of ROS but is quickly converted to $\mathrm{H}_{2} \mathrm{O}_{2}$ spontaneously or by the enzyme superoxide dismutase (SOD) [6]. $\mathrm{H}_{2} \mathrm{O}_{2}$ is not an inherently reactive compound. However, $\mathrm{H}_{2} \mathrm{O}_{2}$ can be transformed into highly reactive and deleterious products: (i) the interactions of $\mathrm{H}_{2} \mathrm{O}_{2}$ with $\mathrm{O}_{2}{ }^{--}$or with trace levels of transition metals can lead to the formation of hydroxyl radicals $\left(\mathrm{HO}^{*}\right.$ ) [7]; (ii) myeloperoxidase (MPO), a hydrogen 
peroxide oxidoreductase that is specifically found in mammalian granulocytic leukocytes, including neutrophils, monocytes, basophils, and eosinophils, contributes considerably to the bactericidal capabilities of these cells via formation of $\mathrm{HOCl}$ from $\mathrm{H}_{2} \mathrm{O}_{2}$ and chlorine ions [8].

Although the responses of neutrophils to different stimulating conditions and pathogens are generally beneficial for host defense, they can be deleterious to the organism if these cells are inappropriately activated. In this sense, overproduction of free radicals and proteolytic enzymes used as defenses against bacteria and microorganisms can be highly toxic to surrounding cells and tissues [9].

Dipyrone (metamizol), aminopyrine (4-dimethylaminoantipyrine; aminophenazone), isopropylantipyrine (propyphenazone), and antipyrine (phenazone) (Fig. 1), are pyrazolone derivatives, pertaining to the first groups of compounds used as analgesic, antipiretic, and antiinflammatory therapeutical drugs [10-12], antipyrine being first prepared in 1883 [10]. The therapeutic activity of antipyrine was increased by an introduction of an isopropyl group on C-4 to produce isopropylantipyrine, which improved the antipyretic and analgesic properties, while maintaining the antiinflammatory activity [10]. The introduction of a dimethylamino group on C-4 of the antipyrine molecule resulted in aminopyrine (4-dimethylaminoantipyrine) [10]. A disadvantage of aminopyrine is it relative insolubility in water. The search for more soluble compounds led to the production of the sodium salt of antipyrinylmethylaminomethane-sulfonic acid (dipyrone) [10].

The pharmacological mechanism of action of pyrazolones is not completely understood, but it is known that it involves the inhibition of cycloxygenase isoenzymes, platelet tromboxane synthesis, and prostanoids synthesis [13-15]. Unfortunately, in several cases, the use of pyrazolones, more specifically of dipyrone and aminopyrine, has been followed

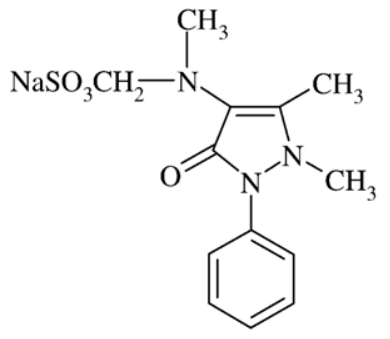

Dipyrone

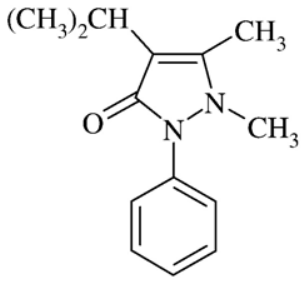

Isopropylantipyrine

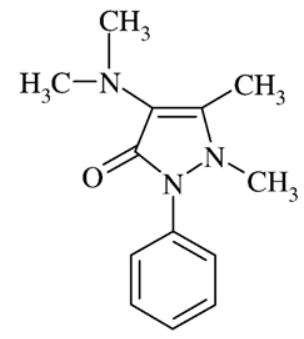

Aminopyrine

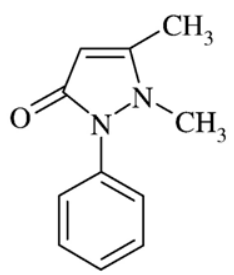

Antipyrine
Fig. 1. Chemical structure of dipyrone, aminopyrine, isopropylantipyrine, and antipyrine. by a severe decrease in the number of circulating neutrophils (agranulocytosis) [15-17]. Because of the risk of agranulocytosis, pyrazolones have been banned or withdrawn from the market in various countries. Nevertheless, these drugs are still available in some countries in Europe including Germany, France, Spain, and Portugal [18]. Pyrazolones are also still widely used in many parts of the world, including the Far East, Africa, and Latin and South America [19], and have been found in some herbal pain remedies (the use of which was also reported to cause agranulocytosis) [20]. Annual sales figures of pyrazolones amount to kilotons, mainly dipyrone and propyphenazone [13]

The absolute risk of agranulocytosis associated with the use of pyrazolone drugs at therapeutical doses and for short periods of time has been considered to be very low [17]. Nevertheless, several studies found strong evidence indicating that the mechanism of pyrazolone-induced agranulocytosis involves the oxidation of these compounds into a reactive intermediate, which then reacts with neutrophils [16]. The possible liability of pyrazolones under prooxidant conditions requires an extensive study on the reactivity of these drugs with neutrophil-produced ROS. On the other hand, little attention has been devoted to the possible hindrance of neutrophil burst by pyrazolones. Such an effect could be beneficial in the case of overactivation of neutrophils, but it can also be highly detrimental if the number of circulating neutrophils is already low. Thus, having in mind the possible importance of pyrazolone reactivity with neutrophil-produced ROS and the pyrazolone-induced hindrance of neutrophil burst, the aim of the present study was to evaluate the putative scavenging activity of the above-mentioned pyrazolones against $\mathrm{O}_{2}{ }^{--}$, $\mathrm{H}_{2} \mathrm{O}_{2}, \mathrm{HO}^{*}$, ROO', and $\mathrm{HOCl}$ and their inhibitory effect against phorbol-12-myristate 13-acetate (PMA)-induced neutrophil burst.

\section{Materials and methods}

\section{Materials}

Dipyrone, aminopyrine, antipyrine, hypoxanthine, xanthine oxidase grade I from buttermilk (EC 1.1.3.22), tiron, ethylenediaminetetraacetic acid (EDTA) disodium salt, ascorbic acid, sodium hypochlorite solution with $4 \%$ available chlorine, lipoic acid, luminol, lucigenin, ferrous chloride, phorbol 12-myristate 13-acetate, histopaque-1077, histopaque-1119, Trypan blue, diphenyleneiodonium chloride (DPI), superoxide dismutase (SOD) (EC 1.15.1.1), Dulbecco's phosphate-buffered saline, without $\mathrm{Ca}^{2+}$ and $\mathrm{Mg}^{2+}$ (PBS), and myeloperoxidase from human leukocytes (EC 1.11.1.7) were obtained from Sigma Chemical Co. (St. Louis, MO, USA). Trolox, dimethyl sulfoxide (DMSO), Tris (hydroxymethyl)aminomethane (Tris buffer), and $\alpha, \alpha^{\prime}$-azodiisobutyramidine dihydrochloride (AAPH) were obtained from Fluka Chemie GmbH (Steinheim, Germany). Fluorescein sodium salt was obtained from Aldrich (Steinheim, Germany). Hydrogen peroxide (30\% solution), sodium carbonate, disodium hydrogen phosphate, and sodium chloride were obtained from Merck (Darmstadt, Germany). 4- 
Aminobenzoic acid hydrazide (ABAH) was obtained from Calbiochem (San Diego, CA, USA). Isopropylantipyrine was a gift from Novartis International Pharmaceutical Ltd. (Ireland).

\section{Measurement of ROS scavenging activity using in vitro} noncellular systems

All measurements were performed in a microplate reader (Synergy HT; BIO-TEK), with fluorimetric or chemiluminescence detection.

\section{Measurement of $\mathrm{O}_{2}^{--}$scavenging activity}

The $\mathrm{O}_{2}{ }^{--}$scavenging activity was measured by chemiluminescence, by monitoring the $\mathrm{O}_{2}{ }^{-}$elicited oxidation of lucigenin, according to a described procedure [21,22] with modifications. $\mathrm{O}_{2}{ }^{--}$are generated by an hypoxanthine/xanthine oxidase system. This system can be used because none of the studied compounds inhibits xanthine oxidase within the tested concentrations. Reaction mixtures contained, in a final volume of $250 \mu \mathrm{L}$, the following reagents at the indicated final concentrations: carbonate buffer $(0.5 \mathrm{M}$; pH 10), lucigenin $(154 \mu \mathrm{M})$, hypoxanthine $(0.4 \mathrm{mM})$, tested compounds dissolved in buffer at various concentrations $(0-5 \mathrm{mM})$, and xanthine oxidase $(150 \mathrm{mU} / \mathrm{mL})$. The resulting chemiluminescence was followed for $5 \mathrm{~min}$. The effects are expressed as the percentage inhibition of the $\mathrm{O}_{2}{ }^{--}$-elicited lucigenin oxidation. Tiron was used as a positive control. Each study corresponds to four experiments performed in triplicate.

\section{Measurement of $\mathrm{H}_{2} \mathrm{O}_{2}$ scavenging activity}

The $\mathrm{H}_{2} \mathrm{O}_{2}$ scavenging activity was measured by chemiluminescence, by monitoring the $\mathrm{H}_{2} \mathrm{O}_{2}$-induced oxidation of lucigenin, according to a described procedure [23]. Reaction mixtures contained, in a final volume of $250 \mu \mathrm{L}$, the following reagents at the indicated final concentrations: Tris-HCl buffer $(50 \mathrm{mM}$; $\mathrm{pH} 7.4)$, lucigenin $(3 \mathrm{mM})$, tested compounds dissolved in buffer at various concentrations $(0-5$ $\mathrm{mM})$, and $\mathrm{H}_{2} \mathrm{O}_{2}(2 \%)$. The resulting chemiluminescence was followed for $5 \mathrm{~min}$. The effects are expressed as the percentage inhibition of the $\mathrm{H}_{2} \mathrm{O}_{2}$-induced lucigenin oxidation. Ascorbic acid and catalase were used as positive controls. Each study corresponds to four experiments performed in triplicate.

\section{Measurement of $\mathrm{HO}^{\bullet}$ scavenging activity}

The $\mathrm{HO}^{\bullet}$ scavenging activity was measured by chemiluminescence, by monitoring the $\mathrm{HO}^{-}$-elicited oxidation of luminol, according to a described procedure [24] with modifications. $\mathrm{HO}^{*}$ were generated by a Fenton system $\left(\mathrm{FeCl}_{2}-\mathrm{EDTA}-\mathrm{H}_{2} \mathrm{O}_{2}\right)$. Reaction mixtures contained, in a final volume of $250 \mu \mathrm{L}$, the following reagents at the indicated final concentrations: carbonate buffer $(0.5 \mathrm{M}$; $\mathrm{pH} 10)$, luminol $(20 \mu \mathrm{M}), \mathrm{FeCl}_{2}$-EDTA $(25 \mu \mathrm{M}, 100 \mu \mathrm{M})$, tested compounds dissolved in buffer at various concentrations $(0-15 \mu \mathrm{M})$, and $\mathrm{H}_{2} \mathrm{O}_{2}(3.5 \mathrm{mM})$. The iron salt was premixed with the chelator dissolved in water before addition to the reaction mixture. This assay was performed at $37^{\circ} \mathrm{C}$. The resulting chemiluminescence was followed for $5 \mathrm{~min}$. The scavenging effects are expressed as the percentage inhibition of the $\mathrm{HO}^{\circ}$-elicited luminol oxidation. Trolox was used as a positive control. Each study corresponds to four experiments performed in triplicate.

\section{Measurement of $\mathrm{HOCl}$ scavenging activity}

The $\mathrm{HOCl}$ scavenging activity was measured by chemiluminescence, by monitoring the $\mathrm{HOCl}$-elicited oxidation of luminol, according to a described procedure [25] with modifications. $\mathrm{HOCl}$ was prepared immediately before use, by adjusting the $\mathrm{pH}$ of a $1 \%(\mathrm{v} / \mathrm{v})$ solution of $\mathrm{NaOCl}$ to 6.2 with diluted sulfuric acid. The concentration of $\mathrm{HOCl}$ was further determined spectrophotometrically at $235 \mathrm{~nm}$ using the molar absorption coefficient of $100 \mathrm{M}^{-1} \mathrm{~cm}^{-1}$ [26]. Reaction mixtures contained, in a final volume of $250 \mu \mathrm{L}$, the following reagents at the indicated final concentrations: phosphate buffer (50 mM; pH 12), luminol $(250 \mu \mathrm{M})$, tested compounds dissolved in buffer at various concentrations $(0-5 \mathrm{mM})$, and $\mathrm{HOCl}(25 \mu \mathrm{M})$. This assay was performed at $37^{\circ} \mathrm{C}$. The resulting chemiluminescence was followed for $10 \mathrm{~s}$. The scavenging effects are expressed as the percentage inhibition of the HOCl-induced luminol oxidation. Lipoic acid was used as a positive control. Each study corresponds to four experiments performed in triplicate.

\section{Measurement of ROO* scavenging activity}

$\mathrm{ROO}^{\bullet}$ scavenging activity was measured by monitoring the fluorescence decay as result of $\mathrm{ROO}^{-}$-induced oxidation of fluorescein, according to a described procedure [27] known as the oxygen radical absorbance capacity (ORAC) assay. ROO' are generated by AAPH in a microplate reader at $37^{\circ} \mathrm{C}$. Reaction mixtures contained, in a final volume of $200 \mu \mathrm{L}$, the following reagents at the indicated final concentrations: phosphate buffer (75 mM; pH 7.4), fluorescein $(61 \mathrm{nM})$, tested compounds dissolved in buffer at various concentrations $(0-8 \mu \mathrm{M})$, and AAPH $(19 \mathrm{mM})$. Fluorescence measurements were performed on a microplate reader with excitation and emission wavelengths of 485 and $528 \mathrm{~nm}$, respectively. The effects are expressed as the relative trolox equivalent ORAC value, which is calculated by the equation: Relative ORAC Value $=\left[\left(\mathrm{AUC}_{\text {sample }}-\mathrm{AUC}_{\text {blank }}\right) /\right.$ $\left(\mathrm{AUC}\right.$ trolox $\left.\left.-\mathrm{AUC}_{\text {blank }}\right)\right] \times$ (molarity of trolox/molarity of the compound), where AUC is the area under curve [28]. Ascorbic acid was used as a positive control. Each study corresponds to four experiments performed in triplicate.

\section{Measurement of PMA-induced oxidative burst in human neutrophils}

This study was performed using neutrophils isolated from healthy human volunteers. Essentially, the study was based on the stimulation of human neutrophils with PMA with the subsequent measurement of the neutrophil-ROS-generating 
capacity. For this purpose, chemiluminescence methods, using luminol and lucigenin as probes, were adapted to a microplate reader.

As described below, the detection of ROS was evaluated by using ROS-induced oxidation of luminol as the measured endpoint while the specific detection of $\mathrm{O}_{2}^{\cdot-}$ was evaluated by using $\mathrm{O}_{2}{ }^{-}-$induced oxidation of lucigenin as the measured endpoint.

Isolation of neutrophils. Venous blood was collected from healthy human volunteers by antecubital venipuncture, into EDTA vacuum tubes. Whole venous blood was separated into cell types using histopaque solutions 1077 and 1119 in polypropylene 12-mL centrifuge tubes, as reported before [29], with modifications. Briefly, $3 \mathrm{~mL}$ of histopaque 1077 was carefully layered on top of $3 \mathrm{~mL}$ of histopaque 1119 in a 12-mL polypropylene tube. Then $6 \mathrm{~mL}$ of the collected blood was decanted on top of this discontinuous density gradient. The tube was centrifuged at $800 \mathrm{~g}$ for $30 \mathrm{~min}$ at $20^{\circ} \mathrm{C}$. Once the centrifugation was complete the neutrophils were carefully removed using a Pasteur pipette. The neutrophil pellet was removed and doubled in volume using PBS (this reduces the viscosity of the histopaque-neutrophil suspension so that the cells can be centrifuged without the need for high $g$ forces); the neutrophils were centrifuged at $700 \mathrm{~g}$ for $5 \mathrm{~min}$ at $4^{\circ} \mathrm{C}$. The supernatant was decanted and a mixture of $1.25 \mathrm{~mL}$ of PBS $+5.25 \mathrm{~mL}$ of sterile distilled water was added to the neutrophil pellet to lyse any remaining red blood cells. The tube was gently inverted for $1 \mathrm{~min}$ after which isotonicity was reestablished by adding $2.2 \mathrm{~mL}$ of $3 \% \mathrm{NaCl}$. This suspension was then submitted to a new centrifugation at $700 \mathrm{~g}$ for $5 \mathrm{~min}$ at $4^{\circ} \mathrm{C}$ after which the supernatant was decanted and the neutrophil pellet resuspended in PBS. The obtained cell suspensions contained more than $99 \%$ of neutrophils and the control of their viability showed more than $95 \%$ of the cells excluding Trypan blue (0.4\%). Isolated neutrophils were kept in ice until use. The neutrophils were used from one volunteer per experiment.

Oxidation of luminol by neutrophil-generated ROS. The measurement of neutrophil burst was measured by chemiluminescence, by monitoring ROS-induced oxidation of luminol, according to a previously described procedure [30], with modifications. Reaction mixtures contained, in a final volume of $200 \mu \mathrm{L}$, the following reagents at the indicated final concentrations: neutrophils (final suspension $=10^{6}$ cells $/ \mathrm{mL}$ ), tested compounds at various concentrations $(0-2 \mathrm{mM})$, luminol $(500 \mu \mathrm{M})$, and PMA $\left(1.6 \times 10^{-7} \mathrm{M}\right)$. Cells and tested compounds were preincubated for $5 \mathrm{~min}$ at $37^{\circ} \mathrm{C}$ before the addition of luminol and PMA. The measurements were carried out at $37^{\circ} \mathrm{C}$. Kinetic readings were initiated immediately after cell stimulation. Measurements were taken at the peak of the curve. In all experiments, this peak was observed around $5 \mathrm{~min}$. The effects are expressed as the percentage inhibition of the luminol oxidation. Lipoic acid was used as a positive control. Each study corresponds to four experiments performed in triplicate.

Oxidation of lucigenin by neutrophil-generated $\mathrm{O}_{2}{ }^{--}$. The measurement of neutrophil burst was measured by chemilu- minescence, by monitoring $\mathrm{O}_{2}^{--}$induced oxidation of lucigenin, according to a previously described procedure [30], with modifications. Reaction mixtures contained, in a final volume of $200 \mu \mathrm{L}$, the following reagents at the indicated final concentrations: neutrophils (final suspension $=5 \times 10^{6}$ cells/ $\mathrm{mL})$, tested compounds at various concentrations $(0-2 \mathrm{mM})$, lucigenin $(125 \mu \mathrm{M})$, and PMA $\left(1.6 \times 10^{-7} \mathrm{M}\right)$. The measurements were carried out at $37^{\circ} \mathrm{C}$. Kinetic readings were initiated immediately after cell stimulation. Measurements were taken at the peak of the curve. In all experiments, this peak was observed around $5 \mathrm{~min}$. The effects are expressed as the percentage inhibition of lucigenin oxidation. Tiron, DPI, and SOD were used as positive controls. Each study corresponds to four experiments performed in triplicate.

\section{Luminol oxidation by $\mathrm{HOCl}$ generated by purified myeloperoxidase}

The oxidation of luminol by purified-MPO-generated $\mathrm{HOCl}$ was performed according to a previously described procedure [31], with modifications. Reaction mixtures contained, in a final volume of $250 \mu \mathrm{L}$, the following reagents at the indicated final concentrations: $\mathrm{NaCl}(100$ $\mathrm{mM})$, luminol $(250 \mu \mathrm{M})$, tested compounds at various concentrations $(0-2 \mathrm{mM}), \mathrm{MPO}(0.5 \mathrm{U} / \mathrm{mL})$, and $\mathrm{H}_{2} \mathrm{O}_{2}$ $(100 \mu \mathrm{M})$. Kinetic readings were initiated immediately after addition of $\mathrm{H}_{2} \mathrm{O}_{2}$. All measurements were carried out at constant temperature of $37^{\circ} \mathrm{C}$, and were taken at the peak of the curve. In all experiments, this peak was observed around 7 to $9 \mathrm{~min}$. The inhibitory effects of the tested compounds are expressed as the percentage inhibition of the luminol oxidation. ABAH, a MPO inhibitor, was used as positive control. Each study corresponds to four experiments performed in triplicate.

\section{Results}

$\mathrm{O}_{2}^{\cdot-}$ scavenging activity

No scavenging activity was observed for any of the tested nonsteroidal antiinflamatory drugs (NSAIDs) at concentrations up to $5 \mathrm{mM}$. An $\mathrm{IC}_{50}$ of $3.7 \pm 0.7 \mu \mathrm{M}$ (mean $\pm \mathrm{SEM}$ ) was found for the positive control, tiron (Table 1).

\section{$\mathrm{H}_{2} \mathrm{O}_{2}$ scavenging activity}

No scavenging activity was observed for any of the tested NSAIDs at concentrations up to $5 \mathrm{mM}$. $\mathrm{IC}_{50 \mathrm{~s}}$ of $1035 \pm 33 \mu \mathrm{M}$ (Table 1) and $50 \pm 5 \mathrm{U} / \mathrm{mL}$ (mean $\pm \mathrm{SEM}$ ) were found for the positive controls ascorbic acid and catalase, respectively.

\section{$H^{\bullet}$ scavenging activity}

Fig. 2 shows the results obtained in the $\mathrm{HO}^{\bullet}$ scavenging assay. Dipyrone, aminopyrine, isopropylantipyrine, and antipyrine prevented $\mathrm{HO}^{\circ}$-induced luminol oxidation in a concen- 
Table 1

Scavenging activities ( $\mathrm{IC}_{50} \mu \mathrm{M}$, mean \pm SEM) of antipyrine, isopropylantipyrine, aminopyrine, and dipyrone and the positive controls trolox, tiron, ascorbic acid, and lipoic acid, against $\mathrm{O}_{2}{ }^{-}, \mathrm{H}_{2} \mathrm{O}_{2}, \mathrm{HO} \mathrm{H}^{\bullet}$, and $\mathrm{HOCl}$

\begin{tabular}{|c|c|c|c|c|}
\hline \multirow[t]{2}{*}{ Tested compound } & \multicolumn{4}{|l|}{$\underline{\mathrm{IC}_{50}(\mu \mathrm{M})}$} \\
\hline & $\mathrm{O}_{2}^{\bullet-}$ & $\mathrm{H}_{2} \mathrm{O}_{2}$ & $\mathrm{HO}^{\bullet}$ & $\mathrm{HOCl}$ \\
\hline Antipyrine & NA & NA & $>5000$ & $>5000$ \\
\hline Isopropylantipyrine & NA & NA & $2393 \pm 424$ & $1557 \pm 167$ \\
\hline Aminopyrine & NA & NA & $2.1 \pm 0.4$ & $20.2 \pm 2.5$ \\
\hline Dipyrone & NA & NA & $5.8 \pm 0.5$ & $4.5 \pm 0.7$ \\
\hline Trolox & - & - & $0.70 \pm 0.07$ & - \\
\hline Tiron & $3.7 \pm 0.7$ & - & - & - \\
\hline Ascorbic acid & - & $1035 \pm 33$ & - & - \\
\hline Lipoic acid & - & - & - & $6.0 \pm 0.5$ \\
\hline
\end{tabular}

NA, no activity was found within the tested concentrations (up to $5000 \mu \mathrm{M}$ ).

tration-dependent manner. Dipyrone and aminopyrine were much more potent scavengers than antipyrine and isopropylantipyrine. The evaluated $\mathrm{IC}_{50} \mathrm{~s}$ were $5.8 \pm 0.5,2.1 \pm 0.4$, $2393 \pm 424$, and $>5000 \mu \mathrm{M}$ (mean \pm SEM) for dipyrone, aminopyrine, isopropylantipyrine, and antipyrine, respectively (Table 1). An $\mathrm{IC}_{50}$ of $0.70 \pm 0.07 \mu \mathrm{M}$ (mean $\pm \mathrm{SEM}$ ) was found for the positive control, trolox.
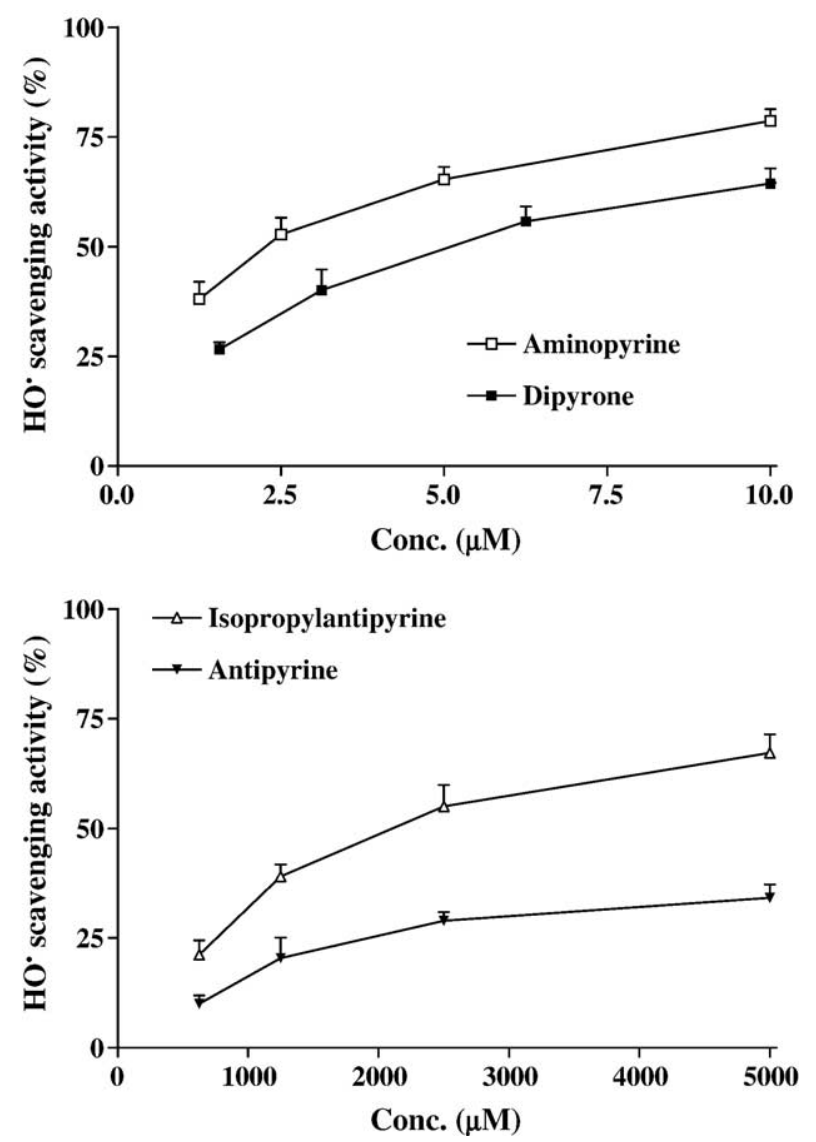

Fig. 2. HO` scavenging activity of dipyrone, aminopyrine, isopropylantipyrine, and antipyrine as determined by their ability to prevent $\mathrm{HO}^{\circ}$-elicited oxidation of luminol. Each point represents the values obtained from four experiments performed in triplicate (mean \pm SEM).

\section{HOCl scavenging activity}

Fig. 3 shows the results obtained in the $\mathrm{HOCl}$ scavenging assay. Dipyrone, aminopyrine, isopropylantipyrine, and antipyrine prevented the $\mathrm{HOCl}$-induced oxidation of luminol in a concentration-dependent manner. In accordance with the results obtained in the $\mathrm{HO}^{\circ}$ scavenging assay, dipyrone and aminopyrine were also much more potent scavengers than antipyrine and isopropylantipyrine. The evaluated $\mathrm{IC}_{50} \mathrm{~s}$ were $4.5 \pm 0.7,20.2 \pm 2.5,1557 \pm 167$, and $>5000 \mu \mathrm{M}$ (mean \pm SEM) for dipyrone, aminopyrine, isopropylantipyrine, and antipyrine, respectively (Table 1 ). An $\mathrm{IC}_{50}$ of $6.0 \pm 0.5 \mu \mathrm{M}$ (mean \pm SEM) was found for the positive control, lipoic acid.

\section{$\mathrm{ROO}^{\bullet}$ scavenging activity}

Table 2 shows the results obtained in the $\mathrm{ROO}^{\bullet}$ scavenging assay. Again, the results obtained in this study demonstrate that $\mathrm{ROO}^{*}$ is effectively scavenged by dipyrone and aminopyrine, while antipyrine is much less active and isopropylantipyrine is devoid of any activity against this ROS (ORAC values are $2.4 \pm 0.3,1.3 \pm 0.3$, and $0.08 \pm 0.01$ (mean \pm SEM) for dipyrone, aminopyrine, and antipyrine, respectively) (Table
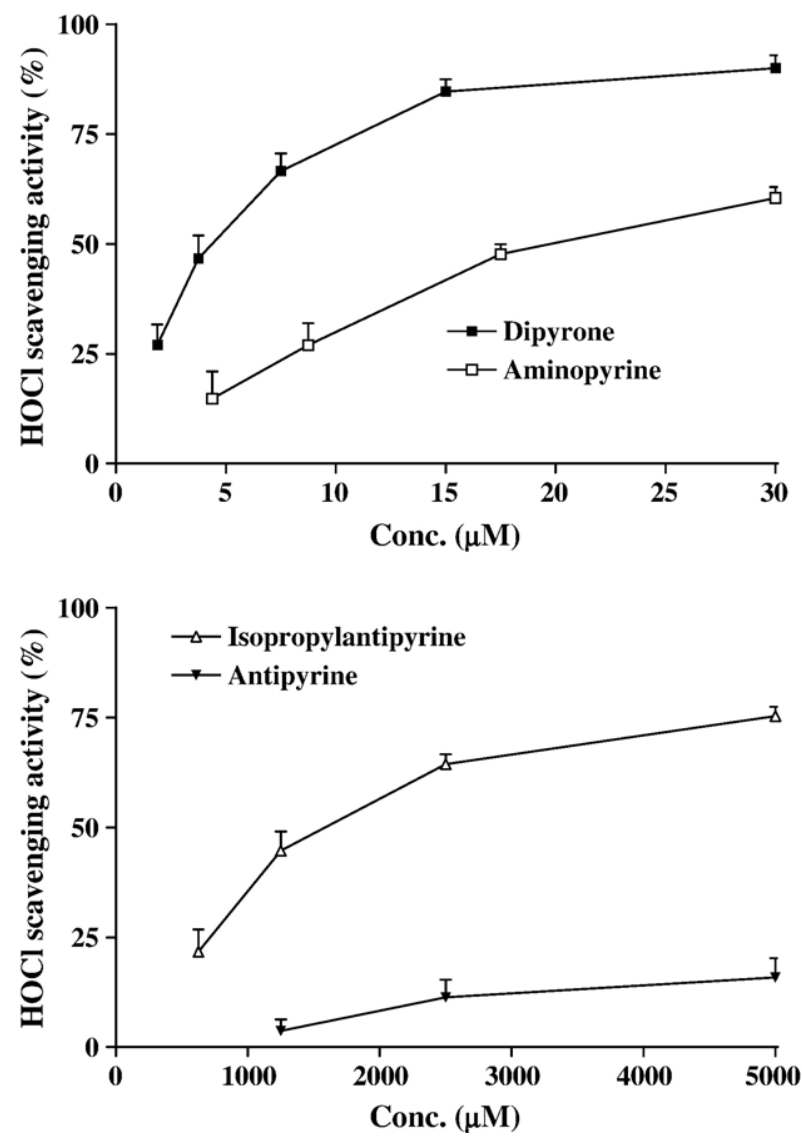

Fig. 3. HOCl scavenging activity of dipyrone, aminopyrine, isopropylantipyrine, and antipyrine as determined by their ability to prevent HOCl-elicited oxidation of luminol. Each point represents the values obtained from four experiments performed in triplicate (mean \pm SEM). 
Table 2

$\mathrm{ROO}^{\bullet}$ scavenging activity (mean \pm SEM) of antipyrine, isopropylantipyrine, aminopyrine, dipyrone, and ascorbic acid, as measured by monitoring the fluorescence decay in result of $\mathrm{ROO}^{\circ}$-induced oxidation of fluorescein, known as oxygen radical absorbance capacity (ORAC) assay

\begin{tabular}{ll}
\hline Tested compound & $\begin{array}{l}\mathrm{ORAC}_{\mathrm{ROO}} \pm \mathrm{SEM} \\
(\mu \mathrm{M} \text { trolox equivalents/ } \mu \mathrm{M} \text { compound })\end{array}$ \\
\hline Antipyrine & $0.08 \pm 0.01$ \\
Isopropylantipyrine & $\mathrm{NA}$ \\
Aminopyrine & $1.31 \pm 0.29$ \\
Dipyrone & $2.37 \pm 0.31$ \\
Ascorbic acid & $0.25 \pm 0.07$ \\
\hline
\end{tabular}

Values are presented as the relative potency to the positive control trolox. NA, no activity was found.

2). The ORAC value found for the positive control ascorbic acid was $0.25 \pm 0.07$ (mean $\pm \mathrm{SEM}$ ).

\section{Inhibition of neutrophil burst}

The measurement of neutrophil burst was performed in two complementary assays. In the first assay the neutrophil burst was measured by chemiluminescence, by monitoring ROSinduced oxidation of luminol (Fig. 4). It can be observed that all the tested compounds, except antipyrine, were effective inhibitors of the neutrophil burst, dipyrone being the most active. Aminopyrine was also highly effective in preventing the neutrophil burst, at the same concentrations as dipyrone, but isopropylantipyrine displayed only weak inhibitory activity, yet
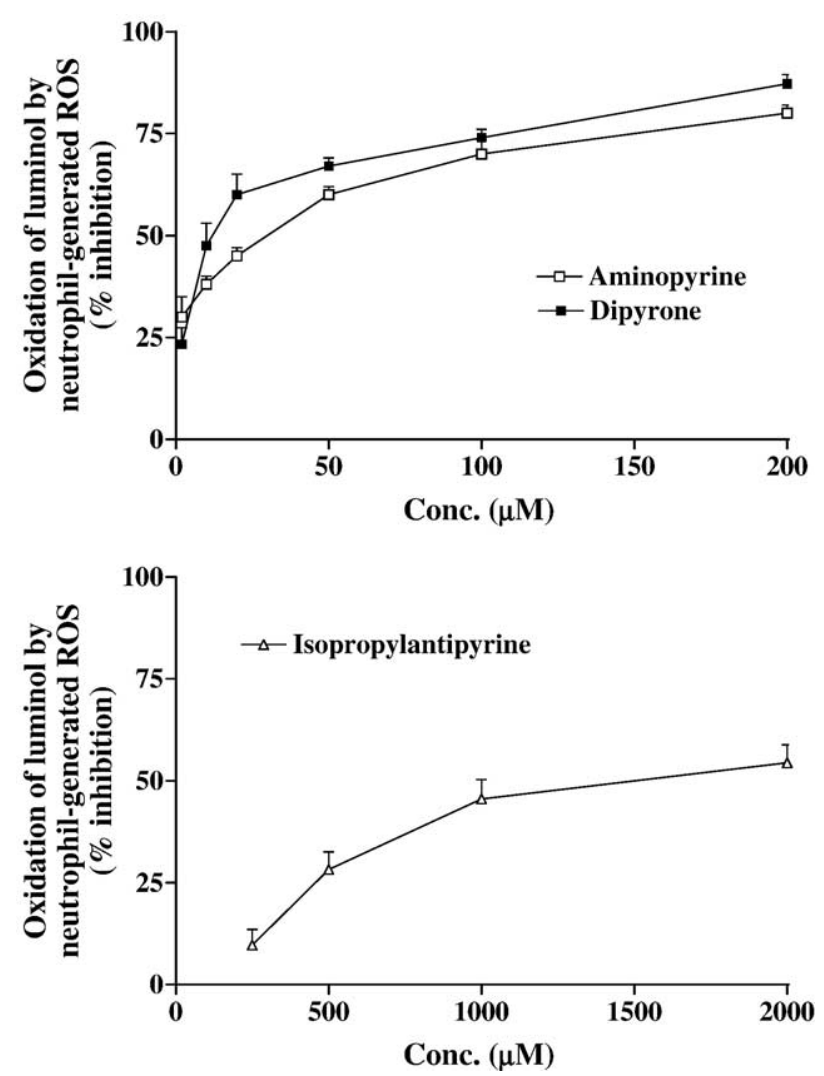

Fig. 4. Inhibition (\%) of neutrophil-generated ROS-induced oxidation of luminol by dipyrone, aminopyrine, and isopropylantipyrine. Each point represents the values obtained from four experiments performed in triplicate (mean $\pm \mathrm{SEM}$ ).
Table 3

Inhibition of neutrophil burst by antipyrine, isopropylantipyrine, aminopyrine, dipyrone, diphenyleneiodonium chloride (DPI), tiron, lipoic acid, 4-aminobenzoic acid $(\mathrm{ABAH})\left(\mathrm{IC}_{50} \mu \mathrm{M}\right.$, mean $\left.\pm \mathrm{SEM}\right)$, and superoxide dismutase (SOD) $\left(\mathrm{IC}_{50} \mathrm{U} / \mathrm{mL}\right.$, mean $\left.\pm \mathrm{SEM}\right)$

\begin{tabular}{llll}
\hline Tested compound & \multicolumn{1}{l}{$\mathrm{IC}_{50}(\mu \mathrm{M})$} & \\
\cline { 2 - 3 } & $\begin{array}{l}\text { Neutrophils- } \\
\text { luminol }\end{array}$ & $\begin{array}{l}\text { Neutrophils- } \\
\text { lucigenin }\end{array}$ & $\begin{array}{l}\mathrm{MPO}- \\
\text { luminol }\end{array}$ \\
\hline Antipyrine & $\mathrm{NA}$ & $\mathrm{NA}$ & $\mathrm{NA}$ \\
Isopropylantipyrine & $1410 \pm 427$ & $\mathrm{NA}$ & $\mathrm{NA}$ \\
Aminopyrine & $25.0 \pm 4.0$ & $\mathrm{NA}$ & $33.3 \pm 3.0$ \\
Dipyrone & $13.4 \pm 3.5$ & $\mathrm{NA}$ & $5.6 \pm 0.3$ \\
DPI & - & $1.1 \pm 0.1$ & - \\
Tiron & - & $88.0 \pm 10.0$ & - \\
Lipoic acid & $943 \pm 271$ & - & - \\
ABAH & - & - & $0.34 \pm 0.01$ \\
SOD $(\mathrm{U} / \mathrm{mL})$ & - & $59 \pm 4$ & - \\
\hline
\end{tabular}

NA, no activity was found within the tested concentrations (up to $2000 \mu \mathrm{M}$ ).

still similar to that of lipoic acid. $\mathrm{IC}_{50}$ values were $13.4 \pm 3.5$, $25.0 \pm 4.0$, and $1410 \pm 427 \mu \mathrm{M}$ (mean \pm SEM) for dipyrone, aminopyrine, and isopropylantipyrine, respectively, while the $\mathrm{IC}_{50}$ values found for the positive control lipoic acid was $943 \pm$ $271 \mu \mathrm{M}$ (mean \pm SEM) (Table 3).

In a second assay, the neutrophil burst was also measured by chemiluminescence, by monitoring $\mathrm{O}_{2}{ }^{--}$induced oxidation of lucigenin. In this assay no inhibitory activity was found for the tested compounds up to the concentration of $2000 \mu \mathrm{M}$. The $\mathrm{IC}_{50}$ values found for the positive controls DPI (inhibitor of NADPH oxidase), tiron (scavenger of $\mathrm{O}_{2}{ }^{-}$), and SOD were $1.1 \pm 0.1 \mu \mathrm{M}, 88.0 \pm 10.0 \mu \mathrm{M}$, and $59 \pm 4 \mathrm{U} / \mathrm{mL}$ (mean $\pm \mathrm{SEM}$ ), respectively (Table 3 ).

\section{Luminol oxidation by MPO-generated $\mathrm{HOCl}$}

Fig. 5 shows the results obtained in the oxidation of luminol by MPO-generated $\mathrm{HOCl}$. In this assay effective activity was

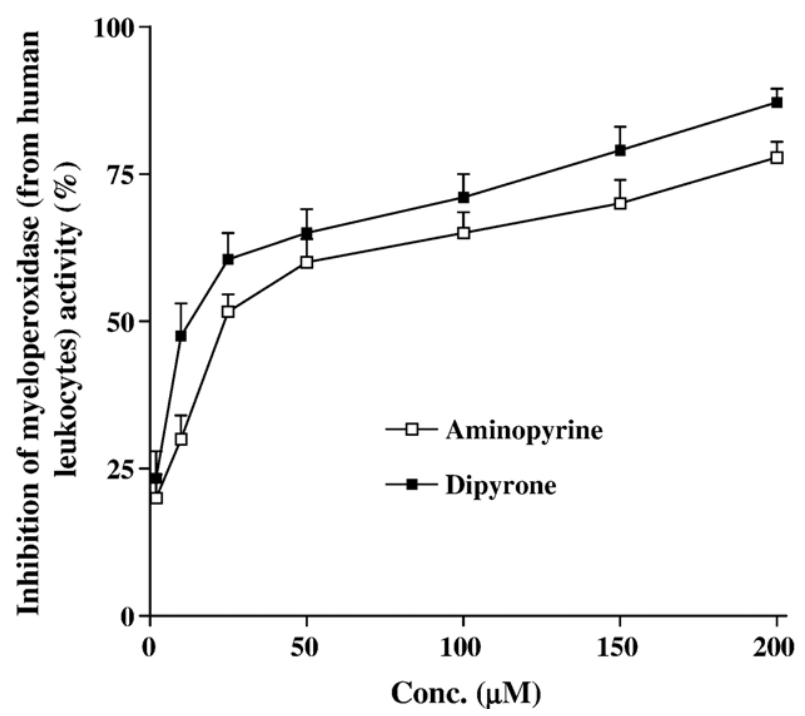

Fig. 5. Inhibition (\%) of luminol oxidation by myeloperoxidase (from human leukocytes)-generated $\mathrm{HOCl}$ by dipyrone and aminopyrine. Each point represents the values obtained from four experiments performed in triplicate $($ mean \pm SEM) 
observed only for dipyrone and aminopyrine, which, nevertheless, were shown to be highly potent compounds, with $\mathrm{IC}_{50}$ values of $5.6 \pm 0.3$ and $33.3 \pm 3.0 \mu \mathrm{M}$ (mean $\pm \mathrm{SEM}$ ), respectively (Table 3 ). The $\mathrm{IC}_{50}$ value found for the positive control ABAH was $0.34 \pm 0.01 \mu \mathrm{M}$ (mean $\pm \mathrm{SEM}$ ) (Table 3).

\section{Discussion}

The results obtained in the present study confirm that aminopyrine and dipyrone prevent PMA-induced neutrophil burst with high efficiency, while isopropylantipyrine had little effect and antipyrine had no effect at all. In general, the inhibition of neutrophil burst can be due either to the inhibition of ROS-generating enzymes or to the direct ROS-scavenging effect. Taking into consideration that the main ROS produced by neutrophils are NADPH-oxidase-generated $\mathrm{O}_{2}{ }^{--}$(with subsequent formation of $\mathrm{H}_{2} \mathrm{O}_{2}, \mathrm{HO}^{\circ}$, and $\left.\mathrm{ROO}^{\circ}\right)[3,6]$ and MPOgenerated $\mathrm{HOCl}$ [8], it was of great interest to evaluate the scavenging effect of the studied pyrazolones against these species. It was clearly shown that aminopyrine and dipyrone were highly potent scavengers of $\mathrm{HO}^{*}$ and $\mathrm{HOCl}^{\circ}$, while, in accordance with the neutrophil burst results, isopropylantipyrine had little effect and antipyrine showed only a residual effect against these two ROS. None of the studied pyrazolones was capable of scavenging $\mathrm{O}_{2}^{--}$or $\mathrm{H}_{2} \mathrm{O}_{2}$, while dipyrone was shown to be the most reactive against ROO'.

Most of the neutrophil burst and purified human MPO assays, including those performed in the present study, use the oxidation of luminol or lucigenin as the measured endpoints. While luminol can be oxidized by several ROS [30], lucigenin is oxidized mainly by $\mathrm{O}_{2}{ }^{--}$[30] or $\mathrm{H}_{2} \mathrm{O}_{2}$ [23]. With regard to the direct effect of the studied NSAIDs on the activity of MPO purified from human leucocytes, it was demonstrated that aminopyrine and particularly dipyrone prevented the MPOinduced oxidation of luminol with high efficacy. Importantly, aminopyrine was previously shown to have a strong inhibitor effect against MPO, while antipyrine and 4-aminoantipyrine had no effect [32]. Thus, it may be postulated that the observed scavenging effects of aminopyrine and dipyrone against $\mathrm{HO}^{\circ}$ and $\mathrm{HOCl}$, together with the direct MPO inhibition by aminopyrine, should contribute to the prevention of neutrophil burst and MPO-induced oxidation of luminol. This was confirmed by the observed lack of inhibitory effects when the oxidation of lucigenin was the measured endpoint. Indeed, in this case, no inhibitory effect was observed for any of the tested pyrazolones, which is in agreement with the absence of $\mathrm{O}_{2}{ }^{--}$scavenging activity observed for these compounds. These last results also indicate that none of the studied compounds have NADPH oxidase inhibitor effects.

The observed reactivity of pyrazolones against neutrophilproduced ROS may also help to understand their possible contribution to agranulocytosis. Two major types of agranulocytosis induced by drugs have been described, one of an immunological type, which is caused by drug-induced antibodies, and another of a toxic type, where the offending agent drug and/or its metabolites suppress cell production in the bone marrow [33]. With regard to the pyrazolones tested in the present work, aminopyrine has long been associated with a high incidence of agranulocytosis [16] and is the best-characterized drug with regard to its chemical and biological reactivity. Indeed, several studies found strong evidence indicating that the mechanism of aminopyrine-induced agranulocytosis involves a drug-dependent antineutrophil antibody that requires covalent binding of the drug, or, most probably, a reactive derivative of the drug, to neutrophils [16]. The generation of an aminopyrine-reactive derivative can be accomplished through its oxidation. In the present study, it was observed that aminopyrine and dipyrone are highly reactive with $\mathrm{HO}^{\circ}$, $\mathrm{ROO}^{\circ}$, and $\mathrm{HOCl}$. The oxidation of aminopyrine by neutrophil-MPO-generated $\mathrm{HOCl}$ was previously reported, leading to the formation of a blue cation radical $\left(\mathrm{AP}^{\cdot+}\right)$, to which has been attributed the potentially fatal bone marrow toxicity, leading to agranulocytosis [34,35]. It has been proposed that the mechanism by which $\mathrm{HOCl}$ oxidizes aminopyrine to a cation radical involves N-chlorination followed by loss of a chlorine radical [35]. Another possible mechanism is the formation of a dication by the loss of chloride ion from $\mathrm{N}$ chloroaminopyrine [16]. This intermediate is very reactive, with a half-life of approximately $15 \mathrm{~ms}$, and, in addition to being the precursor of the cation radical, it also appears to react with two molecules of water to form several other products that are consistent with the proposed dication intermediate [16]. Thus, according to Uetrecht et al. [16], the reactive dication formed by neutrophil-derived $\mathrm{HOCl}$ could also be responsible for aminopyrine-induced agranulocytosis.

The formation of $\mathrm{AP}^{{ }^{+}}$has also been shown to be mediated by other enzymatic systems, namely by prostaglandin $\mathrm{H}$ synthase [36], lipoxygenase [37], and horseradish peroxidase $[38,39]$. Peroxidase-catalyzed aminopyrine metabolism involves two $\mathrm{N}$-demethylation steps which release $\mathrm{HCHO}$ and form 4-methylaminoantipyrine and then aminoantipyrine via intermediate N-cation radicals [40]. Of note, GSH prevents aminopyrine metabolism and $\mathrm{HCHO}$ release by reducing the aminopyrine N-cation radical to aminopyrine [41]. Furthermore, NADH is also oxidized by aminopyrine radicals to form $\mathrm{NAD}^{+}$, which is accompanied by oxygen uptake and formation of reactive oxygen species [40]. Importantly, aminoantipyrine, a metabolite of aminopyrine and dipyrone [15,42], was found to be more effective than aminopyrine at catalyzing GSH or NADH oxidation [40], which contributes to the reported toxic effect of these parent compounds.

Dipyrone is itself a prodrug that undergoes nonenzymatic hydrolysis in the stomach to form 4-methylaminoantipyrine, which is rapidly and almost completely absorbed [15,42]. Therefore, the agranulocytosis effect that has been attributed to dipyrone $[15,17,18]$ is in accordance with the above-mentioned chemical and biological reactivity, with $\mathrm{N}$-chlorination of this metabolite, followed by loss of a chlorine radical to form a cation radical [40].

The attribution of agranulocytosis to antipyrine and isopropylantipyrine is only anecdotal. However, antipyrine is a $\mathrm{CH}$-acidic compound that readily undergoes electrophilic substitution at the 4 position, hence giving potential chemical and biological reactivity to this molecule [43]. An acute 
allergic reaction to antipyrine was already reported [44]. 4Isopropylantipyrine is also mentioned as elicitor of allergic/ pseudoallergic reactions, for which only scarce data on the nature of the underlying mechanisms are available [45]. It was recently demonstrated that immediate-type adverse reactions to 4-isopropylantipyrine could be classified as Type I allergy and are mediated by specific IgE antibodies [45]. Of note, a common IgE-binding epitope for pyrazolone drugs was previously described [46].

Although the absolute risk of agranulocytosis associated with the use of pyrazolones has been considered to be very low, their reactivity with neutrophil-derived $\mathrm{HO}^{\circ}, \mathrm{ROO}^{\circ}$, and $\mathrm{HOCl}$ with possible formation of reactive intermediates capable of inducing agranulocytosis should be taken into account. Also, the hindrance of neutrophil burst by dipyrone and aminopyrine may be important. As mentioned before, the MPO inhibitor effect, together with the scavenging activity against $\mathrm{HO}^{\circ}, \mathrm{ROO}^{\circ}$, and $\mathrm{HOCl}$, could be beneficial in the case of overactivation of neutrophils. However, these effects can also be highly detrimental if the number of circulating neutrophils is already decreased. In this sense, aminopyrine and dipyrone were the most reactive, while antipyrine and isopropylantipyrine were demonstrated to be the less reactive compounds. On the other hand, these two latter pyrazolones may also be endowed with potential adverse effects, as judged by their allergenicity.

\section{Acknowledgments}

The authors acknowledge the financial support given by FCT and FEDER in the ambit of the project POCTI/FCB/ 47186/2002. David Costa acknowledges FCT and FSE his PhD grant (SFRH/BD/10483/2002).

\section{References}

[1] Swain, S. D.; Rohn, T. T.; Quinn, M. T. Neutrophil priming in host defense: role of oxidants as priming agents. Antioxid. Redox Signal. 4:69-83; 2002

[2] Zhang, X.; Kluger, Y.; Nakayama, Y.; Poddar, R.; Whitney, C.; DeTora, A.; Weissman, S. M.; Newburger, P. E. Gene expression in mature neutrophils: early responses to inflammatory stimuli. J. Leukoc. Biol. 75:358-372; 2004

[3] Quinn, M. T.; Gauss, K. A. Structure and regulation of the neutrophil respiratory burst oxidase: comparison with nonphagocyte oxidases. J. Leukoc. Biol. 76:760-781; 2004.

[4] Weiss, S. J. Tissue destruction by neutrophils. N. Engl. J. Med. 320: $365-376 ; 1989$.

[5] Yang, K. D.; Hill, H. R. Neutrophil function disorders: pathophysiology, prevention, and therapy. J. Pediatr. 119:343-354; 1991.

[6] Babior, B. M. NADPH oxidase. Curr. Opin. Immunol. 16:42-47; 2004.

[7] Halliwell, B.; Gutteridge, J. M. The importance of free radicals and catalytic metal ions in human disease. Mol. Aspect. Med. 8:89-193; 1985.

[8] Xia, Y.; Zweier, J. L. Measurement of myeloperoxidase in leukocytecontaining tissues. Anal. Biochem. 245:93 -96; 1997.

[9] Pelletier, M.; Roberge, C. J.; Gauthier, M.; Vandal, K.; Tessier, P. A.; Girard, D. Activation of human neutrophils in vitro and dieldrin-induced neutrophilic inflammation in vivo. J. Leukoc. Biol. 70:367-373; 2001.

[10] Brogden, R. N. Pyrazolone derivatives. Drugs 32:60-70; 1986.

[11] Coersmeier, C.; Wittenberg, H. R.; Aehringhaus, U.; Dreyling, K. W.; Peskar, B. M.; Brune, K.; Peskar, B. A. Effect of anti-inflammatory and analgesic pyrazoles on arachidonic acid metabolism in isolated heart and gastric mucosa preparations. Agents Actions Suppl. 19:137-153; 1986.

[12] Gursoy, A.; Demirayak, S.; Capan, G.; Erol, K.; Vural, K. Synthesis and preliminary evaluation of new 5-pyrazolinone derivatives as analgesic agents. Eur. J. Med. Chem. 35:359-364; 2000.

[13] Levy, M. Hypersensitivity of pyrazolones. Thorax 55:S72-S74; 2000.

[14] Graham, G. G.; Scott, K. F. Mechanisms of action of paracetamol and related analgesics. Inflammopharmacology 11:401-413; 2003.

[15] Bentur, Y.; Cohen, O. Dipyrone overdose. J. Toxicol. Clin. Toxicol. 42:261-265; 2004.

[16] Uetrecht, J. P.; Ma, H. M.; MacKnight, E.; McClelland, R. Oxidation of aminopyrine by hypochlorite to a reactive dication: possible implications for aminopyrine-induced agranulocytosis. Chem. Res. Toxicol. 8:226-233; 1995

[17] Ibáñez, L.; Vidal, X.; Ballarín, E.; Laporte, J. R. Agranulocytosis associated with dipyrone (metamizol). Eur. J. Clin. Pharmacol. 60: $821-829 ; 2005$.

[18] Hedenmalm, K.; Spigset, O. Agranulocytosis and other blood dyscrasias associated with dipyrone (metamizol). Eur. J. Clin. Pharmacol. 58: 265-274; 2002

[19] Chan, T. Y. K.; Chan, A. W. K. Aminopyrine-induced blood dyscrasiasStill a problem in many parts of the world. Pharmacoepidemiol. Drug Saf. 5:215-219; 1996.

[20] Ries, C. A.; Sahud, M. A. Agranulocytosis caused by Chinese herbal medicines. Dangers of medications containing aminopyrine and phenylbutazone. J. Am. Med. Assoc. 231:352-355; 1975.

[21] Oosthuizen, M. M. J.; Engelbrecht, M. E.; Lambrechts, H.; Greyling, D.; Levy, R. D. The effect of $\mathrm{pH}$ on chemiluminescence of different probes exposed to superoxide and singlet oxygen generators. J. Biolumin. Chemilumin. 12:277-284; 1997.

[22] Oosthuizen, M. M. J.; Greyling, D. Antioxidants suitable for use with chemiluminescence to identify oxyradical species. Redox Rep. 4:277-290; 1999

[23] Costa, D.; Gomes, A.; Reis, S.; Lima, J. L. F. C.; Fernandes, E. Hydrogen peroxide scavenging activity by non-steroidal anti-inflammatory drugs. Life Sci. 76:2841-2848; 2005.

[24] Oosthuizen, M. M. J.; Greyling, D. Hydroxyl radical generation: the effect of bicarbonate, dioxygen and buffer concentration on $\mathrm{pH}-$ dependent chemiluminescence. Redox Rep. 6:105-116; 2001.

[25] Yildiz, G.; Demiryurek, A. T.; Sahin-Erdemli, I.; Kanzik, I. Comparison of antioxidant activities of aminoguanidine, methylguanidine and guanidine by luminol-enhanced chemiluminescence. $\mathrm{Br}$. J. Pharmacol. 124:905-910; 1998

[26] Aruoma, O. I. Scavenging of hypochlorous acid by carvedilol and ebselen in vitro. Gen. Pharmac. 28:269-272; 1997.

[27] Fernandes, E.; Costa, D.; Toste, S. A.; Lima, J.L.F.C.; Reis, S. In vitro scavenging activity for reactive oxygen and nitrogen species by nonsteroidal anti-inflammatory indole, pyrrole, and oxazole derivative drugs. Free Radic. Biol. Med. 37:1895-1905; 2004.

[28] Huang, D.; Ou, B.; Hampsch-Woodill, M.; Flanagan, J. A.; Prior, R. L. High-throughput assay of oxygen radical absorbance capacity (ORAC) using a multichannel liquid handling system coupled with a microplate fluorescence reader in 96-well format. J. Agric. Food Chem. 50:4437-4444; 2002.

[29] Bland, E. J.; Keshavarz, T.; Bucke, C. Using 2',7'-dichlorodihydrofluorescein-diacetate to assess polysaccharides as immunomodulating agents. Mol. Biotechnol. 19:125-131; 2001.

[30] Hasegawa, H.; Suzuki, K.; Nakaji, S.; Sugawara, K. Analysis and assessment of the capacity of neutrophils to produce reactive oxygen species in a 96-well microplate format using lucigenin and luminol dependent chemiluminescence. J. Immunol. Methods 210:1-10; 1997.

[31] Razumovitch, J. A.; Fuchs, D.; Semenkova, G. N.; Cherenkevich, S. N. Influence of neopterin on generation of reactive species by myeloperoxidase in human neutrophils. Biochim. Biophys. Acta 1672:46-50; 2004.

[32] Kettle, A. J.; Winterbourn, C. C. Mechanism of inhibition of myeloperoxidase by anti-inflammatory drugs. Biochem. Pharmacol. 41: 1485-1492; 1991 
[33] Patton, W.; Dufful, S. Idiosyncratic drug-induced haematological abnormalities. Drug Saf. 11:445-462; 1994.

[34] Kalyanaraman, B.; Sohnle, P. G. Generation of free radical intermediates from foreign compounds by neutrophil-derived oxidants. J. Clin. Investig. 75:1618-1622; 1985.

[35] Sayo, H.; Saito, M. The mechanism of myeloperoxidase-catalysed oxidation of aminopyrine. Xenobiotica 9:957-965; 1990.

[36] Eling, T. E.; Mason, R. P.; Sivarajah, K. The formation of aminopyrine cation radical by the peroxidase activity of prostaglandin $\mathrm{H}$ synthase and subsequent reactions of the radical. J. Biol. Chem. 260:1601-1607; 1985.

[37] Pérez-Gilabert, M.; Sanchez-Ferrer, A.; Garcia-Carmona, F. Oxidation of aminopyrine by the hydroperoxidase activity of lipoxygenase: a new proposed mechanism of $\mathrm{N}$-demethylation. Free Radic. Biol. Med. 23:548-555; 1997.

[38] Griffin, B. W. Free radical intermediate in the N-demethylation of aminopyrine by horseradish peroxidase-hydrogen peroxide. FEBS Lett. 74:139-143; 1977.

[39] Griffin, B. W.; Ting, P. L. Mechanisms of N-demethylation of aminopyrine by hydrogen peroxide catalysed by horseradish peroxidase. Biochemistry 17:2206-2211; 1978 .
[40] Galati, G.; Tafazoli, S.; Sabzevari, O.; Chan, T. S.; O’Brien, P. J. Idiosyncratic NSAID drug induced oxidative stress. Chem. Biol. Interact. 142:25-41; 2002

[41] Moldeus, P.; O'Brien, P. J.; Thor, H.; Berggren, M.; Orrenius, S. Oxidation of glutathione by free radical intermediates formed during peroxidase-catalysed N-demethylation reactions. FEBS Lett. 162: $411-415 ; 1983$.

[42] Volz, M.; Kellner, H. M. Kinetics and metabolism of pyrazolones (propyphenazone, aminopyrine and dipyrone). Br. J. Clin. Pharmacol. 10:299S-308S; 1980.

[43] Roth, H. J. Pharmacokinetic and biotransformation of pyrazolinones. Agents Actions Suppl. 19:205-221; 1986.

[44] McCrea, J. B.; Vlasses, P. H.; Rocci, M. L. Jr. Allergic reaction to antipyrine, a marker of hepatic enzyme activity. DICP 23:38-40; 1989.

[45] Himly, M.; Jahn-Schmid, B.; Pittertschatscher, K.; Bohle, B.; Grubmayr, K.; Ferreira, F.; Ebner, H.; Ebner, C. IgE-mediated immediate-type hypersensitivity to the pyrazolone drug propyphenazone. J. Allergy Clin. Immunol. 111:882-888; 2003

[46] Schneider, C. H.; Kasper, M. F.; De Weck, A. L.; Rolli, H.; Angst, D. Diagnosis of antibody-mediated drug allergy. Allergy 42:597-603; 1987. 